\title{
La matriz de consistencia: una metodología de investigación para desarrollar el estado del arte para emprendimientos artesanales enfocados en las TIC's
}

\section{The consistency matrix: a research methodology to develop the state of the art for craft enterprises focused on ICT's}

\author{
Irma Abrigo Córdova \\ Nirma Mancero Acosta \\ Alexis Hurtado Armijos \\ Paola Jaramillo Castro \\ Universidad Internacional del Ecuador, Ecuador \\ Autor para correspondencia: irabrigoco@uide.edu.ec,nimanceroac@uide.edu.ec, \\ alhurtadoar@uide.edu.ec,pajaramilloca@uide.edu.ec \\ Fecha de recepción: 20 de julio de 2018 - Fecha de aceptación: 30 de agosto de 2018
}

Resumen: La propuesta se originó en el sector artesanal de "Plaza Matilde", concretamente en la primera etapa del proyecto, en la cual se evidenció el escaso conocimiento sobre el plan de negocios e inadecuada aplicación de las Tic's en los emprendimientos artesanales, producto de la falta de capacitación en esta área del conocimiento, hasta el momento, no han recibido cursos relacionados al manejo de las tecnologías para sus actividades comerciales. Con la información recogida en los grupos focales y encuestas, surge la segunda etapa del proyecto, con el que se plantea el uso de la matriz de consistencia como metodología de investigación para desarrollar el estado del arte para emprendimientos artesanales enfocados en las TIC's, siendo la finalidad, obtener de manera precisa los temas adecuados para la construcción del mismo. La matriz de consistencia es un instrumento de gran utilidad, permite evaluar el grado de coherencia y conexión lógica entre el título, problema, objetivos, hipótesis, variables, dimensiones, método, diseño de investigación, población y muestra de estudio. Para el presente caso se ha utilizado hasta las dimensiones en vista que el objetivo es emplearla para la construcción del estado del arte, cabe señalar, para desarrollarlo mediante el instrumento mencionado, se requiere realizar una exhaustiva consulta bibliográfica en: libros, artículos científicos, tesis doctorales, etcétera; siendo imprescindible contar con un buen dominio del tema. Con los resultados obtenidos en la matriz de consistencia, se da lugar a una tercera etapa del proyecto, como es la elaboración de guías para la capacitación del sector artesanal, mismo que se efectuará en los meses de julio y agosto del presente, es importante mencionar que en la actualidad ya se está trabajando en su producción.

Palabras clave: matriz de consistencia; metodología; estado del arte; emprendimiento; Tic's

Abstract: The proposal originated in the artisanal sector of "Plaza Matilde", specifically in the first stage of the project, which evidenced the scant knowledge about the business plan and inadequate application of Tic's in the artisan undertakings, product of The lack of training in this area of knowledge, so far, have not received courses related to the management of technologies for their commercial activities. With the information gathered in the focus groups and surveys, the second stage of the project arises, with which the use of the consistency matrix is proposed as a research methodology to develop the state of the art for craft enterprises focused on ICT's, being the purpose, to obtain in a precise way the suitable subjects for the construction of the same one. The consistency matrix is a very useful instrument, allowing to assess the degree of coherence and logical connection between the title, problem, objectives, hypothesis, variables, dimensions, method, research design, population and study sample. For the present case has been used up 
to the dimensions in view that the goal is to use it for the construction of the state of the art, it should be noted, to develop it through the aforementioned instrument, it is required to make an exhaustive bibliographical reference in: books, scientific articles, thesis doctorals, etc .; being essential to have a good command of the subject. With the results obtained in the consistency matrix, there is a third stage of the project, such as the preparation of guides for the training of the artisanal sector, which will take place in the months of July and August of this year, it is important to mention that is currently working on its production. Key Words: matrix of consistency; methodology; state of the art; entrepreneurship; Tic's

\section{Introducción}

La presente investigación tiene como propósito utilizar la matriz de consistencia para el desarrollo del estado del arte en los emprendimientos artesanales enfocados en las TIC's, es un instrumento de gran importancia en la investigación, puesto que permite evaluar el grado de coherencia y relación lógica entre el título, problema, objetivos, hipótesis, variables y dimensiones; criterios necesarios para construir un buen estado del arte orientado a la elaboración de guías para la capacitación de las participantes.

Se constituye en una propuesta innovadora que permite describir con precisión los contenidos necesarios que serán utilizados en el estado del arte, cabe señalar, que para realizar este procedimiento se debe haber consultado en diferentes fuentes bibliográficas como son: textos, artículos científicos, tesis doctorales, etc.

La propuesta se encuentra conformada por las siguientes secciones: revisión de literatura, objetivos, metodología, resultados, discusión y conclusiones, a continuación, detalladas.

\section{Revisión de la literatura}

Matriz de consistencia: Es un instrumento formado por columnas y filas, permite evaluar el grado de coherencia y conexión lógica entre el título, el problema, los objetivos, las hipótesis, las variables, el tipo, método, diseño de investigación, la población y muestra de estudio (Marroquín, 2012).

La matriz de consistencia presentadas por (Caballero, 2014), (Ríos \& Torres, 2013) y (Carrasco, 2018) permite ubicar de manera didáctica los contenidos del estado del arte.

La matriz de consistencia, según (Carrasco, 2018) es:

Un instrumento valioso que consta de un cuadro formado por columnas y filas; permite consolidar los elementos clave de todo el proceso de investigación, además posibilita evaluar el grado de coherencia y conexión lógica entre el título, el problema, la hipótesis, los objetivos, las variables, el diseño de investigación seleccionado, los instrumentos de investigación, así como la población y la muestra de estudio.

\section{Estado del arte}

La primera etapa que debe desarrollarse dentro de una investigación, es la construcción del estado del arte, pues permite determinar la forma como ha sido tratado el tema, cómo se encuentra el avance de su conocimiento en el momento de realizar una investigación y cuáles son las tendencias existentes, en ese momento cronológico, para el desarrollo de la temática que se va a abordar. (Londoño, Maldonado, \& Calderón, 2014) 
En este apartado (Molina Montoya, 2005) expresa que,

...el origen del estado del arte se remonta a la década de los ochenta, cuando se dirigieron fundamentalmente estudios en el área de las ciencias sociales en América Latina, los cuales procuraban la compilación de la información disponible sobre un tópico determinado, con el objetivo de fundamentar políticas y alternativas de acción para el desarrollo social; de aquí surgió el estado del arte asociado con la finalidad de hacer un reconocimiento de la investigación en la región latinoamericana.

(Medina Montoya, 2004) define al estado del arte como una investigación cualitativa con un enfoque hermenéutico, que tiene el propósito de interpretar el material bibliográfico, mientras que a la investigación documental la concibe como una revisión sistemática del material para contextualizar el objeto de estudio.

Por su parte, (Londoño, Maldonado, \& Calderón, 2014), establecen que la elaboración del estado del arte es,

...una etapa en los procesos de investigación convencionales, como se presenta en los manuales de metodología de la investigación científica. En este sentido, se pueden identificar dos procesos generales: a) la búsqueda, selección, organización y disposición de fuentes de información para un tratamiento racional; b) la integración de la información a partir del análisis de los mensajes contenidos en las fuentes, que corresponde a la dimensión hermenéutica del proceso, muestra los conceptos básicos unificadores. En consecuencia, un estado del arte estudia una porción substancial de la literatura y fuentes relevantes de información en un área y desarrolla un proceso de comprensión que converge en una visión global e integradora y en una comunicación de este resultado para otros.

Para entender el estado del arte, (Gómez Vargas, Galeano Higuita, \& Jaramillo Muñoz, 2015) manifiestan que es necesario comprender el concepto desde lo planteado por diferentes autores representativos como son: Hoyos Botero (2000), Galeano Marín y Vélez Restrepo (2002) cuyas teorías afirman que las metodologías de investigación cualitativa son maneras posibles de hacer investigación en ciencias sociales. Una de sus formas es la investigación documental, que incluye la metodología estado del arte.

En base a los objetivos, un estado del arte puede definirse como:

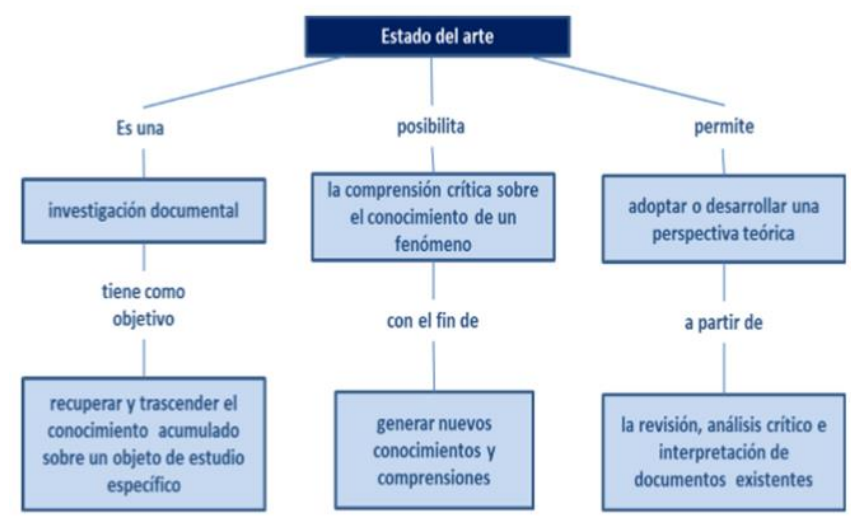

Cuadro 01: Concepto de estado del arte desde los objetivos principales - Autor: (Londoño, Maldonado, \& Calderón, 2014) 


\section{Emprendimiento}

"Un emprendedor es la persona que busca e identifica una oportunidad de negocio, realizando una nueva propuesta de valor para los clientes, y crea o desarrolla un negocio asumiendo sus riesgos inherentes" (Duarte Cueva, 2007).En la literatura hispana, el término es considerado como emprendimiento, empresarismo, emprendurismo y emprendedurismo. Inicialmente entrepreneurship ha sido asimilado a capacidad empresarial, no obstante, se considera un término más extenso, así lo expresa (Toca Torres, 2010)

(Hilarión, 2013) sostiene que el emprendimiento "es el conjunto de actores, instituciones e interrelaciones asociadas con el apoyo que se va emprender, en el cual se especifican los flujos de producto o servicio y el dinero que suceden entre estas instituciones o entidades". Los aspectos que señalan la ruta hacia el desarrollo de la iniciativa emprendedora se agrupan en fuerzas externas y externas, detalladas a continuación. (Silva, 2013)

\begin{tabular}{|c|c|}
\hline \multicolumn{2}{|c|}{ Ruta hacia el desarrollo de la iniciativa emprendedora } \\
\hline Fuerzas internas para afianzar el emprendimiento & Fuerzas externas para estimular el emprendimiento \\
\hline $\begin{array}{ll}\text { - } & \text { Necesidad de realización personal. } \\
\text { - } & \text { Vocación innovadora. } \\
\text { - } & \text { Integridad y responsabilidad social. } \\
\text { - } & \text { Orientación al reconocimiento y las recompensas. } \\
\text { - } & \text { Armonía organizacional. } \\
\text { - } & \text { Autonomía. }\end{array}$ & $\begin{array}{ll}\text { - } & \text { Lo político y lo económico. } \\
\text { - } & \text { El contexto sociocultural. } \\
\text { - } & \text { El contexto tecnológico. } \\
\text { - } & \text { La cultura laboral. } \\
\text { - } & \text { El contexto organizacional. } \\
\text { - } & \text { El contexto familiar. } \\
\text { - } & \text { El contexto educacional. }\end{array}$ \\
\hline
\end{tabular}

Cuadro 02: Ruta hacia el desarrollo de la iniciativa emprendedora.

Fuente. Datos tomados de Emprendedor, hacia un emprendimiento sostenible (2013).

En un emprendimiento se observan las siguientes fases: estudio de mercado, estudio de producción, estudio técnico y estudio financiera, a continuación, descritos.

Estudio de mercado. El marketing se encarga del proceso de planear las actividades de la empresa en relación con el precio, la promoción, la distribución y la venta de bienes y servicios que ofrece, así como la definición del producto o servicio con base en las preferencias del consumidor, de forma tal que permitan crear un intercambio (entre empresa y consumidor) que satisfaga los objetivos de los clientes y de la propia organización. (Alcaraz, 2015).

Aspectos principales de un estudio de mercado. (Morales \& Morales, 2009) establecen los principales aspectos que debe considerar un estudio de mercado son:

- $\quad$ Perfil de los productos y/o servicios.

- $\quad$ Análisis de la oferta.

- $\quad$ Análisis de la demanda.

- $\quad$ Análisis de los precios,

- $\quad$ Análisis los canales de distribución.

- $\quad$ Promoción.

La investigación de mercados es la función que vincula al consumidor, al cliente y al público con la empresa por medio de la información, la cual se utiliza para identificar y definir las oportunidades y los problemas de marketing... (McDaniel \& Gates, 2011). 
El estudio técnico puede subdividirse a su vez en cuatro componentes, que son: determinación del tamaño óptimo de la planta, determinación de la localización óptima de la planta, ingeniería del proyecto y análisis organizativo, administrativo y legal. (Bacan Urbina, 2010).

Estudio administrativo. Para (Morales \& Morales, 2009) la administración es una actividad que se desarrolla en todo tipo de empresas con el objetivo de alcanzar los máximos resultados. Se utiliza una serie de pasos llamado proceso administrativo, el cual comprende las siguientes etapas:

- Previsión.

- Planeación.

- Organización.

- $\quad$ Integración.

- Dirección y

- Control.

Estudio financiero es una síntesis cuantitativa que demuestra con un margen razonable de seguridad, la realización del proyecto con los recursos programados y la capacidad de pago de la empresa. (Ocampo J., 2003).

\section{Las TIC's}

(Filion, Cisneros, \& Mejía-Morelos, 2011) afirman que,

...las tecnologías de la información y comunicación son el conjunto de programas (software), equipo computacional (hardware) y redes de comunicación que se utilizan para procesar y transmitir información. Desde sus inicios a mediados del siglo XX, las TIC se han empleado en el mundo de los negocios, inicialmente para automatizar las operaciones (beneficios operativos) y luego para crear ventajas competitivas (beneficios estratégicos). En medio de un panorama caracterizado por el uso intensivo de la tecnología en el ambiente empresarial, las firmas que no invierten en TIC limitan su posibilidad de crecimiento y ponen en riesgo su viabilidad. Por estas razones, los gobiernos consideran prioritario que las PYMES adopten tecnologías de la información comunicación.

Este conjunto de herramientas permite la obtención y uso de la información a fin de visualizar y entender el medio ambiente, tener un mayor control sobre éste y crear soluciones a problemas y situaciones específicas. (González, y otros, 2010)

\begin{tabular}{llll}
\hline & \multicolumn{2}{c}{ Influencia de las TIC's } \\
El conocimiento & Las profesiones & La organización \\
\hline Porque expande rápidamente & Que ahora demandan un & Al cambiar la forma de hacer negocio y las \\
la visión y estimula la & alto nivel de habilidades en & expectativas culturales sobre lo que ofrece como \\
creatividad en nuevas áreas, a la & los empleados, tanto para el & producto o servicios. En cuanto a su estructura, \\
vez que facilita almacenar, & trabajo que deberán hacer, & se generan organizaciones delgadas y menos \\
desplegar, comunicar y obtener & como en el tipo de trabajo jerárquicas, ya que se forman con menos puestos \\
conocimiento nuevo. & disponible. & y menos niveles organizacionales.
\end{tabular}

Cuadro $\mathrm{N}^{\circ}$ 03: Influencia de las Tecnologías de la Información y Comunicación. Fuente: Datos tomados de tecnologías de la información (2010) 
(Cohen \& Asín, 2014) manifiestan que,

...los sistemas de información y comunicación, mejoran la estructura competitiva del negocio, influyen en el medio ambiente de la organización, por ejemplo, a través de nuevos servicios a clientes, nuevos productos y mercados, adquisiciones de nuevos negocios y oportunidades de inversión. También influyen en el desarrollo del trabajo interno de la organización, ya sea para aumentar la productividad o reducir los costos.

Por otra parte, el papel que las TIC juegan en las empresas también ha experimentado un cambio profundo, pasando de ser simples herramientas de tratamiento de datos, para convertirse en la columna vertebral que afecta a todas las actividades de una organización. (Rey, 2010).

\section{Objetivos}

Determinar la importancia de la matriz de consistencia para la elaboración del estado del arte en los emprendimientos artesanales.

Utilizar la matriz de consistencia como metodología para el desarrollo del estado del arte en los emprendimientos artesanales enfocados en las TIC's.

Desarrollar una nueva propuesta de investigación en base a los resultados del estado del arte trabajado en la matriz de consistencia.

\section{Metodología}

La investigación se realizó a través de un diseño cualitativo e interpretativo, de tipo documental, el cual determinó el procedimiento de selección, acceso y registro de la muestra documental.

\section{Unidades de estudio.}

En la matriz de consistencia se ubicaron los temas hacer abordados para los emprendimientos artesanales enfocados en las TIC's. Previamente se clasificaron en la herramienta Excel 102 documentos como son: libros, trabajos de doctorado, artículos de revista entre otros. Posteriormente se seleccionaron los adecuados para ser utilizados en la literatura del estado del arte.

\section{Instrumento}

Se utilizó la matriz de consistencia la cual facilitó la ubicación detallada de: problemas principales y secundarios, objetivos generales y específicos, hipótesis principal y secundarias, variables; y en relación a ello se realizó la operacionalización de las variables para determinar con precisión los temas para el estado del arte enfocado en las TIC's a ser utilizado en la capacitación que se realizará al sector artesanal de Plaza Matilde.

\section{Procedimiento}

Una vez diseñada la matriz de consistencia, la cual guía con precisión y objetividad el camino a seguir para el desarrollo del trabajo, se accedió a la bibliografía seleccionada para su lectura, revisión y copiado textual de la información requerida, con la finalidad de desarrollar el estado del arte, el cual será empleado en las guías de capacitación para las emprendedoras del sector artesanal de Plaza Matilde. 


\section{Resultados}

Como resultado se presenta la siguiente matriz de consistencia desarrollada en base a las necesidades de las emprendedoras de "Plaza Matilde", previamente a este proceso, se trabajó con grupos focales y se aplicó encuestas para determinar sus requerimientos.

Una vez obtenidas las necesidades de los participantes se procede a trabajar la matriz de consistencia, en la cual se ubican: problemas, objetivos, hipótesis, variables e indicadores; con estos criterios se realiza el estado de arte pertinente para abordar la temática.

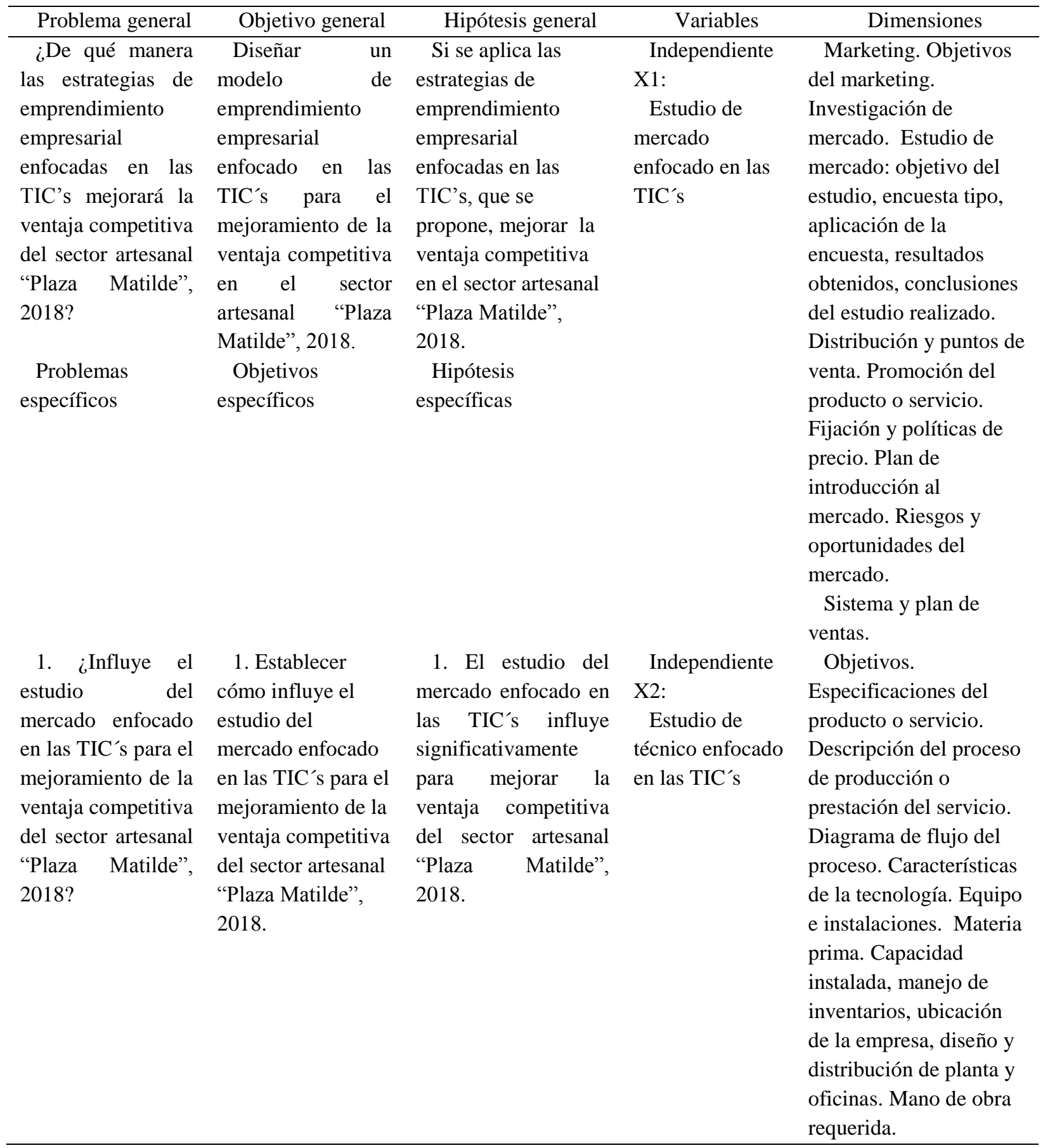




\begin{tabular}{|c|c|c|c|c|}
\hline 2. ¿Impacta el & 2. Evaluar cómo & 2. El estudio técnico & Independiente & Objetivos, estructura \\
\hline estudio técnico & impacta el estudio & enfocado en las TIC's & X3: Estudio de & organizacional. \\
\hline enfocado en las & técnico enfocado en & impacta & administrativo & Funciones específicas \\
\hline $\begin{array}{l}\text { TIC's para el } \\
\text { mejoramiento de la } \\
\text { ventaja competitiva }\end{array}$ & $\begin{array}{l}\text { las TIC's para el } \\
\text { mejoramiento de la } \\
\text { ventaja competitiva }\end{array}$ & $\begin{array}{l}\text { positivamente en el } \\
\text { mejoramiento de la } \\
\text { ventaja competitiva }\end{array}$ & $\begin{array}{l}\text { enfocado en las } \\
\text { TIC's }\end{array}$ & $\begin{array}{l}\text { por puesto. Captación de } \\
\text { personal, desarrollo del } \\
\text { personal, administración }\end{array}$ \\
\hline en el sector & del sector artesanal & del sector artesanal & & de sueldos y salarios. \\
\hline artesanal del "Plaza & "Plaza Matilde", & "Plaza Matilde", 2018 & & Evaluación del \\
\hline Matilde", 2018? & 2018 & & & $\begin{array}{l}\text { desempeño, relaciones } \\
\text { de trabajo y marco legal } \\
\text { de la organización. }\end{array}$ \\
\hline 3. ¿Contribuye el & 3. Determinar & 3. El estudio & Independiente & Sistema contable de la \\
\hline estudio & cómo contribuye el & administrativo & X4: Estudio de & empresa, costos y \\
\hline administrativo & estudio & enfocado en las TIC's & Financiero & gastos. \\
\hline enfocado en las & administrativo & contribuye & enfocado en las & Estados financieros \\
\hline TIC's para mejorar & enfocado en las & significativamente en & TIC's & proyectados. \\
\hline la ventaja & TIC's para el & el mejoramiento de la & & Indicadores financieros. \\
\hline $\begin{array}{l}\text { competitiva del } \\
\text { sector artesanal }\end{array}$ & $\begin{array}{l}\text { mejoramiento de la } \\
\text { ventaja competitiva }\end{array}$ & $\begin{array}{l}\text { ventaja competitiva } \\
\text { del sector artesanal }\end{array}$ & & \\
\hline "Plaza Matilde", & del sector artesanal & "Plaza Matilde", & & \\
\hline $2018 ?$ & "Plaza Matilde", & 2018. & & \\
\hline & 2018 & & & \\
\hline 4. ¿Incide el & 3. Definir como & estudio & Dependiente & Diferenciación de \\
\hline estudio financiero & incide el estudio & financiero enfocado & Y1: v & productos: calidad y \\
\hline enfocado en las & financiero enfocado & en las TIC's incide & competitiva & precios. \\
\hline TIC's al & en las TIC's para el & significativamente en & & \\
\hline mejoramiento de la & mejoramiento de la & el mejoramiento de la & & \\
\hline ventaja competitiva & ventaja competitiva & ventaja competitiva & & \\
\hline del del sector & del sector artesanal & del sector artesanal & & \\
\hline artesanal "Plaza & "Plaza Matilde", & "Plaza Matilde", & & \\
\hline Matilde", 2018? & 2018. & 2018. & & \\
\hline
\end{tabular}

\section{Discusión}

En la primera fase del proyecto se utilizó la metodología cualitativa como observación participante, entrevistas y grupos focales; y la cuantitativa utilizando encuestas a gerentes de los emprendimientos pertenecientes a Plaza Matilde. Las dos metodologías permitieron conocer los requerimientos de las participantes y aplicación de las herramientas tecnológicas en los emprendimientos.

Se evidenció problemas de falta de utilización las TIC's y desconocimiento de un plan de negocios en los emprendimientos, siendo prioritario como siguiente paso, la capacitación del sector artesanal orientada a estas temáticas, en la cual se instruirá sobre las herramientas tecnológicas aplicadas en cada fase de emprendimiento, de esta manera se pretende mejorar la ventaja competitiva de cada negocio.

Para lograr este propósito, en la segunda etapa del proyecto, se planteó utilizar la matriz de consistencia como metodología de investigación para desarrollar el estado del arte para emprendimientos artesanales enfocados en las TIC's; en la misma se ubicó el problema general y 
específicos, el objetivo general y específicos, hipótesis principal y secundarias; así como las variables y dimensiones, estas dos últimas categorías se originaron de las necesidades observadas en las emprendedoras de "Plaza Matilde" y fue el punto de partida para la construcción del estado de arte.

La matriz de consistencia es un instrumento que permitió desarrollar el estado del arte de manera ordenada y objetiva, el cual estuvo en concordancia del título con el problema, objetivo y la hipótesis general; existiendo una correspondencia entre el número de dimensiones de la variable independiente con el número de elementos específicos del problema, objetivo e hipótesis general.

Actualmente con estos resultados se está elaborando una guía de capacitación para las participantes, a ser impartida en los meses de julio-agosto del 2018, de esta manera se estará llegando a la tercera fase del proyecto y por consiguiente concluyendo este proyecto macro en beneficio de las artesanas de "Plaza Matilde" de la ciudad de Loja.

\section{Conclusiones}

La matriz de consistencia es una técnica valiosa para el desarrollo del estado del arte en los emprendimientos artesanales enfocados en las TIC's, puesto que orienta de manera detallada y correcta las temáticas a ser abordadas.

Se determina la importancia de la matriz de consistencia para la elaboración del estado del arte en los emprendimientos artesanales, misma que permitirá la elaboración de guías conducentes a la capacitación del sector artesanal de "Plaza Matilde".

Cabe destacar que se origina una nueva propuesta de investigación en base a los resultados del estado del arte plasmado en la matriz de consistencia.

\section{Bibliografía}

Alcaraz, R. R. (2011). El Emprendedor de Éxito. México, D.F.: Editores S.A. de C.V.

Bacan Urbina, G. (2010). Evaluación de Proyectos. México: Programas Educativos S.A. de C.V.

Caballero, R. A. (2014). Metodología integral innovadora para planes y tesis. La metodología del cómo formularlos. México, D.F.: Cengage Learning Editores S.A. de C.V.

Carrasco, D. S. (2018). Metodología de la investigación científica. Pautas metodológicas para diseñar y elaborar el proyecto de investigación (Segunda ed.). Lima: San Marcos de Aníbal Paredes Galván.

Cohen, D., \& Asín, E. (2014). Tecnologìas de la Información. México: Mexicana, Reg. Nùm.736.

Duarte Cueva, F. (2007). Emprendimiento, empresa y crecimiento empresarial. Contabilidad y Negocios, vol. 2, núm. 3, 46-56.

Filion, L. J., Cisneros, M. L., \& Mejía-Morelos, J. (2011). Administración de PYMES. México D.F.: Litográfica Ingramex S.A.de C.V. 
Gómez Vargas, M., Galeano Higuita, C., \& Jaramillo Muñoz, D. A. (2015). El estado del arte: una metodología de investigación. Revista Colombiana de Ciencias Sociales Vol. 6 No. 2, 423 442 .

González, M., Dolores, L., Mmaría, L., Caballero, M., Valdez, A., Dieck, M., . . María, E. (2010).

Tencnologías de la Informaciòn. Mèxico: Editores, S.A. DE C.V.

Hilarión, J. (2013). Emprendimiento e innovación. México: Artgraph.

Londoño, P. O., Maldonado, G. L., \& Calderón, V. L. (2014). Guía para construir el estado del arte.

International corporation of Networks of Knowledge, 1-39.

Marroquín, P. R. (2012). Matriz operacional de la variable y matriz de consistencia.

McDaniel, C., \& Gates, R. (2011). Investigación de mercados. México D.F.: Cengage Learning Editores S.A.

Medina Montoya, S. I. (2004). Función social del psicólogo clínico: estado del arte. (Trabajo de grado inédito). Medellín: Universidad San Buenaventura.

Molina Montoya, N. P. (2005). ¿Qué es el estado del arte? Ciencia y Tecnología para la salud Visual y Ocular No 5: 73-75, 73-75.

Morales, A., \& Morales, J. A. (2009). Proyectos de inversión. México, D.F.: Edesa Impresiones S.A. de C.V.

Ocampo, J. (2003). Costos y Evaluación de proyectos. México: Marbeth, S.A. de C.V.

Rey, À. G. (2010). Sistemas de Información. Herramientas prácticas para la gestión. México: S.A de C.V.

Ríos, T., \& Torres, T. (2013). La tesis. Lima: UNMSM.

Silva, J. (2013). Emprendedor. Hacia un emprendimiento sostenible. Bogotá: Alfaomega Colombiana S.A.

Toca Torres, C. (2010). Consideraciones para la formación en emprendimiento: explorando nuevos ámbitos y posibilidades. Estudios Gerenciales, 41-60. 